\title{
Core Topics in Endocrinology in Anaesthesia and Critical Care
}

\author{
George M. Hall, Jennifer M. Hunter, Mark S. Cooper. Cambridge University Press, \\ Cambridge, United Kingdom, 2010. ISBN: 978-0-521-50999-2
}

\author{
Manoj M. Lalu, MD, PhD · Roseanne Yeung, MD
}

Published online: 10 August 2011

(C) Canadian Anesthesiologists' Society 2011

Core Topics in Endocrinology in Anaesthesia and Critical Care is a concise and well-written textbook. Its senior editors include two distinguished professors of anesthesia (G.M.H. and J.M.H.), and the third editor (M.S.C.) is an eminent endocrinologist who adds a multidisciplinary scope to the book. In our view, given the high prevalence of endocrine disease, the topics covered in this book are pertinent to most practicing anesthesiologists.

The book is comprised of three sections with six chapters in each section. Section 1, Perioperative care of patients with endocrine disease, includes chapters which address the topics of thyroid, parathyroid, and adrenal disease. Another chapter deals with neuroendocrine tumours, including carcinoid syndrome, a disease whose incidence may be highest on anesthesia licensing exam questions. The final chapter in Section 1 covers the molecular mechanisms and features of multiple endocrine neoplasia.

Section 2, Perioperative care of the patient with diabetes mellitus, is fitting given the dramatic increase in the incidence of this disease. Individual chapters review the perioperative care of diabetic patients in various settings, including ambulatory, pediatric, obstetrical, and surgical inpatient settings. The pharmacological management of this disease has grown increasingly complex, and the editors acknowledge this reality by devoting an entire chapter to an in-depth review of this subject. Although not all

M. M. Lalu, MD, PhD ( $\varangle)$

The Ottawa Hospital University of Ottawa, Ottawa, ON, Canada

e-mail: manojlalu@gmail.com

R. Yeung, MD

University of British Columbia, Vancouver, BC, Canada anesthesiologists will agree with the recommendations in this section, we acknowledge the editors' concerted effort to provide clear instructions on the perioperative management of diabetes

Section 3, Endocrine disorders in the critically ill, begins with a thoughtful review of the endocrine pathophysiology which underlies critical illness; moreover, the information is kept pertinent to the practicing anesthesiologist. The beginning chapter also outlines the difficulties of appropriate management in the dynamic setting of critical illness. Glucose control, glucocorticoids, sodium balance, and thyroid dysfunction are covered in subsequent chapters.

Overall, we found this book to be up-to-date, appropriately succinct, and highly readable. Given its slim form, we were initially surprised by the number of topics the editors chose to cover. However, even with the restricted page length, the most pertinent aspects of each disease were covered and perioperative management was outlined in adequate detail. Thus, the editors should be commended on their ability to summarize this vast field of medicine in an accessible manner.

At times, Core Topics in Endocrinology in Anaesthesia and Critical Care does wade into controversial territory, such as the discussion on glucose management in the critically ill. Earlier trials of tight glucose control demonstrated positive outcomes, and more recent trials found negative outcomes (e.g., NICE-SUGAR), thus, many intensivists approach this topic cautiously. Surprisingly, the authors of this particular chapter support tight glucose control despite contradictory evidence in their own summary which makes "it impossible to set unambiguous recommendations". The authors do provide some qualifications for their support (e.g., computerized protocols for intensive glucose control), and they acknowledge the need for further research. 
One concern, which often arises with multi-author texts, is undue repetition and variable writing among chapters. This textbook does have some inconsistencies among chapters, for instance, case vignettes are presented in some chapters but not in all. Also, in a number of chapters, there are certain statements where we would have welcomed references being cited.

In addition, the authors often delve into the molecular mechanics which orchestrate the endocrine system. For instance, the textbook includes a description of phosphotyrosine phosphatise $1 \mathrm{~B}$ and other molecular mediators of insulin signalling. This level of detail may be esoteric to the average anesthesiologist and will likely not affect daily clinical practice. In fact, some of the molecular mechanisms outlined in this book may be under debate even in the realm of basic science. Given the high level of detail in some sections, we were surprised by the superficial treatment of anesthetic considerations in others. For example, the clinical features and underlying pathology of multiple endocrine neoplasia are discussed in detail, yet the practical perioperative concerns of this disorder are not summarized. Despite this inconsistency, the editors do provide excellent overviews of the anesthetic considerations of endocrine diseases in the majority of the chapters.

Overall, we consider Core Topics in Endocrinology in Anaesthesia and Critical Care a worthy addition to any collection of perioperative medicine textbooks. While brief, it remains highly relevant and could be useful to both students and experienced practitioners alike.

Competing interests None declared. 